Annuaire suisse de politique de développement

13 | 1994

Annuaire Suisse - Tiers Monde 1994

\title{
I. Situation économique en 1992
}

\section{Monika Egger, Gérard Perroulaz et René Longet}

\section{OpenEdition}

\section{Journals}

Édition électronique

URL : http://journals.openedition.org/aspd/1000

DOI : $10.4000 /$ aspd. 1000

ISSN : 1663-9669

\section{Éditeur}

Institut de hautes études internationales et du développement

\section{Édition imprimée}

Date de publication : 1 janvier 1994

Pagination : $3-11$

ISSN : 1660-5934

\section{Référence électronique}

Monika Egger, Gérard Perroulaz et René Longet, «I. Situation économique en 1992 », Annuaire suisse de politique de développement [En ligne], 13 | 1994, mis en ligne le 27 novembre 2012, consulté le 08 septembre 2020. URL : http://journals.openedition.org/aspd/1000 ; DOI : https://doi.org/10.4000/ aspd. 1000

Ce document a été généré automatiquement le 8 septembre 2020

(c) The Graduate Institute I Geneva 


\title{
I. Situation économique en 1992
}

\author{
Monika Egger, Gérard Perroulaz et René Longet
}

\section{Vue d'ensemble}

1 L'année économique 1992 aura été, pour les pays industrialisés, une année de récession plus ou moins marquée. Les relations économiques et commerciales ont par ailleurs été sensibles aux répercussions du changement de système des pays d'Europe de l'Est et de l'ancienne Union soviétique (pays de l'Est) qui passent d'une économie à planification centralisée à une économie de marché ouverte à la concurrence. Ce groupe de pays a connu une croissance extrêmement variable déterminée en bonne partie par la mesure de leur engagement dans les mécanismes du marché libre. Quant au groupe des pays en développement, il a enregistré en 1992 une croissance moyenne positive, mais fort variable selon les régions, le niveau de développement de ses différents membres et leur degré d'insertion dans l'économie mondiale. Ce sont les pays asiatiques qui ont connu la plus forte croissance; les pays d'Amérique latine et centrale ont également connu, dans l'ensemble, une croissance positive. Les pays africains, qui représentent la région économiquement la plus faible de la planète, ont enregistré une croissance absolue minime au cours de l'année 1992 ; leur PIB par habitant a même reculé sous l'effet de la poussée démographique.

2 Le mouvement de création de zones économiques régionales favorisant les échanges s'est poursuivi au cours de l'année 1992 : les pays de la Communauté européenne vont se doter d'un marché Intérieur commun (traité économique européen de Maastricht); les Etats-Unis, le Canada et le Mexique préparent un accord de libre-échange nordaméricain ALENA (NAFTA), quatre pays d'Amérique latine (Argentine, Brésil, Paraguay, Uruguay) projettent de s'unir en un Mercosur. En Asie, les six membres de l'ASEAN (Association of South East Asian Nations: Thaïlande, Malaisie, Singapour, Brunei, Indonésie et Philippines) constituent avec les autres nouvelles économies industrielles de la région et le géant économique en pleine croissance qu'est la Chine une région économique puissante. 
En 1992, les pays de l'OCDE et les six pays économiquement les plus dynamiques d'Asie (Hong Kong, Corée, Malaisie, Singapour, Taiwan et Thaïlande) ont ouvert des consultations sur le renforcement de leurs relations économiques. En 1993, quatre pays d'Amérique latine se sont joints à ces négociations : l'Argentine, le Brésil, le Chili et le Mexique. De nombreux autres petits groupes de pays ont signé des accords commerciaux ou s'efforcent de renforcer les collaborations régionales, comme, par exemple, les sept pays du Sud-Est asiatique que sont l'Inde, le Pakistan, le Bangladesh, le Sri Lanka, le Népal, les Maldives et le Bhoutan, qui projettent de stimuler le commerce régional en supprimant leurs barrières douanières.

4 Ce renforcement du bilatéralisme et du régionalisme va à contresens de l'Uruguay Round, les négociations du GATT, dont l'objectif est de libéraliser le commerce mondial dans un cadre multilatéral.

5 Classification des pays en phase de transition

6 Les réformes en cours dans les pays de l'Est et le développement économique de la zone Asie-Pacifique imposent une redéfinition de la classification des pays. Il s'agit, en particulier, d'y intégrer les statistiques des pays en cours de transition de l'économie planifiée à l'économie de marché et des pays de l'ex-Union soviétique. Nous utilisons pour les classer le terme de pays de l'Est, lequel regroupe donc tous les pays de l'exUnion soviétique et d'Europe de l'Est. Selon la classification de l'OCDE, les cinq pays asiatiques suivants font partie depuis 1993 des pays en développement : le Kazakhstan, le Kirghizistan, l'Ouzbékistan, le Tadjikistan et le Turkménistan. En revanche, le comité du développement de l'OCDE a proposé de retirer de la liste des pays en développement, dans les trois années à compter de janvier 1993, les pays économiquement consolidés que sont Singapour, les Bahamas, le Katar, le Koweït, les Emirats arabes et le Brunei. Pour plus de détails sur l'actuelle classification des pays, nous renvoyons le lecteur aux définitions de l'OCDE et de l'ONU reprises dans la partie statistique.

\section{Développement par région}

8 2.1. Pays industrialisés : les sept principaux pays industrialisés ont enregistré en 1992 une croissance réelle de leur production (PIB) de 1,5\%, les autres de $1 \%$. Les Etats-Unis ont connu une légère relance alors que les pays européens voyaient leur production reculer, leur chômage augmenter et leurs déficits budgétaires se creuser. La récession amorcée en 1989 s'est en général poursuivie en 1992. La Suisse a enregistré en 1992, comme en 1991, un recul de sa production brute, cette fois de 0,6\%. Le taux de chômage y a dépassé $4 \%$. Dans les pays de l'OCDE, le taux moyen de chômage a atteint $8 \%$ en 1992.

2.2. Pays de l'Est : la politique de réforme de ce groupe de pays prévoit, entre autres, les mesures énergiques suivantes: libéralisation des prix et du commerce extérieur, ouverture du pays aux investissements étrangers, adoption de nouveaux taux de change. Les pays d'Europe de l'Est pris dans leur ensemble ont connu en 1992 une forte chute de leur production de l'ordre de $16 \%$, après un recul du PIB d'environ $12 \%$ en 1991 et d'environ $5 \%$ en 1990. Mais la situation économique des pays ayant le plus progressé sur la voie de l'économie de marché (Pologne, Hongrie, ex-Tchécoslovaquie) 
s'est améliorée. Le secteur privé y a connu un fort développement. Ces pays ont réussi à réorienter leurs exportations vers les pays industrialisés et à attirer des investissements étrangers. La restructuration économique y entraîne de profondes réformes politiques et se révèle être un processus de longue haleine marqué par le recul de la production et l'aggravation du chômage qui, pour une bonne part de la population, s'accompagne de dures privations.

10 Ce sont les pays de l'ex-Union soviétique qui, en 1992, ont le moins progressé sur la voie de la stabilité économique. Cela est dû en partie au manque de clarté des réformes politiques et monétaires (effondrement de la zone rouble). De nombreuses républiques ont connu des désordres politiques et des confrontations ayant parfois dégénéré en guerre civile. Les tentatives de redressement de l'économie sont souvent sapées par le poids de la dette extérieure. Tous les pays de ce groupe partagent les problèmes suivants : accroissement des déficits budgétaires, forte dépendance de la croissance des exportations, taux d'intérêts réels élevés et privatisation des anciennes entreprises d'Etat. A quoi s'ajoute, par exemple en Russie, une grave fuite des capitaux ; le manque de devises a empêché le pays d'honorer le service de sa dette.

11 Les pays industrialisés affirment toujours vouloir soutenir le processus de réforme en Europe de l'Est et dans les anciennes républiques soviétiques. C'est ainsi qu'en 1993, le sommet économique de Tokyo faisait une "Déclaration pour un monde plus sûr et plus humain". Les pays concernés jugent toutefois que les actes sont longs à suivre les promesses. Ils critiquent en particulier les barrières que les pays industrialisés ont dressées contre les importations en provenance d'Europe de l'Est afin de protéger leurs propres produits.

12 2.3. Pays en développement: la croissance de la production dans les pays en développement et dans les nouvelles économies industrielles a encore progressé en 1992 pour atteindre 4,6 \%. Mais si l'activité économique s'est montrée vive en Asie et au Proche-Orient, elle s'est affaiblie en Afrique et en Amérique latine. Le tableau 1 présente la croissance globale du PIB réel des pays en développement et des nouvelles économies industrielles.

13 Amérique latine et centrale : les pays d'Amérique latine et centrale ont poursuivi et approfondi leur réforme économique engagée au cours des années 80 . Une politique qui, au début des années 90 , a relancé la production de la plupart de ces pays. Les années 80 , quant à elles, y sont dorénavant considérées comme "la décennie perdue" du développement. Cela, entre autres, à cause du poids de l'endettement et de l'hémorragie des capitaux qui s'en est suivi. Depuis 1991, le flux net des capitaux s'est à nouveau inversé en faveur de ces pays, en particulier sous l'effet du retour des capitaux en fuite et des investissements directs. La croissance du PIB a atteint 2,9\% en 1991 et $2,3 \%$ en 1992. Avec une production en recul, le Pérou et le Brésil font exception à cette évolution. Les facteurs qui ont favorisé la croissance économique de la région sont avant tout internes; il s'agit en particulier de l'augmentation des investissements et de la demande en consommation. La pauvreté dans laquelle s'enfoncent de vastes couches de la population constitue un problème socio-politique brûlant pour l'ensemble de la région. Le succès à long terme de la politique de réforme dépendra de la manière dont le fruit de cette croissance sera réparti: d'une manière équitable pour le bien de l'ensemble de la population, ou alors au seul profit d'une petite élite. 
Tableau $N^{\circ}$ 1. Croissance du PIB dans les pays en développement

\begin{tabular}{|c|c|c|c|c|}
\hline \multirow[t]{3}{*}{ Pays et groupes de pays } & \multicolumn{4}{|c|}{ PIB réels } \\
\hline & $\begin{array}{c}\text { Moyenne } \\
1984-89\end{array}$ & 1990 & 1991 & 1992 \\
\hline & \multicolumn{4}{|c|}{ Variation en $\%$} \\
\hline Afrique & 3,1 & 1,7 & 1,6 & 1,1 \\
\hline Nigeria & 5,8 & 8,2 & 4,5 & 3,6 \\
\hline Afrique du Sud & 1,5 & $-0,5$ & $-0,4$ & $-2,0$ \\
\hline Pays subsahariens & 2,8 & 0,9 & 0,5 & 0,4 \\
\hline Moyen Orient & $-0,5$ & 5,5 & 2,1 & 6,0 \\
\hline Egypte ${ }^{1}$ & 6,9 & 2,6 & 2,3 & 0,7 \\
\hline $\operatorname{Iran}^{2}$ & $-4,1$ & 11,7 & 8,1 & 6,5 \\
\hline Asie & 7,1 & 5,8 & 5,2 & 6,9 \\
\hline Chine & 9,2 & 4,8 & 7,7 & 12,8 \\
\hline Inde ${ }^{1}$ & 6,1 & 5,5 & 1,2 & 4,2 \\
\hline Indonésie & 5,3 & 7,1 & 6,6 & 5,9 \\
\hline Malaisie & 4,5 & 9,8 & 8,7 & 8,0 \\
\hline Philippines & 2,2 & 2,7 & $-0,7$ & 0,0 \\
\hline Thaïlande & 8,6 & 10,0 & 8,2 & 7,4 \\
\hline NEI & 8,6 & 6,8 & 7,1 & 5,3 \\
\hline Amérique latine & 2,4 & 0,7 & 2,9 & 2,3 \\
\hline Argentine & $-1,3$ & 0,0 & 8,9 & 8,7 \\
\hline Brésil & 4,5 & $-4,0$ & 0,9 & $-0,9$ \\
\hline Chili & 6,2 & 2,1 & 6,0 & 10,4 \\
\hline Mexique & 1,0 & 4,4 & 3,6 & 2,6 \\
\hline Pérou & $-0,5$ & $-4,4$ & 2,6 & $-2,7$ \\
\hline Venezuela & 2,0 & 6,9 & 10,4 & 7,3 \\
\hline Ensemble des pays ${ }^{3}$ & 3,7 & 3,7 & 3,5 & 4,6 \\
\hline
\end{tabular}

Sources : FMI, Perspectives de l'économie mondiale ; ONU, Commission économique pour l'Amérique latine et les Caraïbes ; données nationales ; in Rapport annuel BRI, Bâle 1993.

1) année budgétaire

2) année commençant le 21 mars

3) calculs effectués sur la base des pondérations du PIB et des taux de change de 1984-86.

14 Asie : en 1992, la région économiquement la plus dynamique ade nouveau été l'Asie. La Chine se distingue par une croissance réelle du PIB de $13 \%$. Dans les nouvelles économies industrielles, le rythme de la croissance s'est un peu ralenti après avoir fortement accéléré les années précédentes. L'Inde a connu une remarquable relance. Les principaux facteurs de croissance de ces pays sont en général la modestie du coût de la main-d'œuvre, un remarquable renforcement de la productivité ainsi qu'un considérable élargissement du commerce.

15 Afrique : en 1992, la croissance moyenne de la production n'a atteint que $1 \%$. La sécheresse dont une grande partie du continent a souffert, a beaucoup nui à l'agriculture. Les désordres politiques de nombreux pays ont également eu des conséquences désastreuses sur le processus de développement. Le Nigeria (tout comme l'Afrique du Sud) entre pour $20 \%$ dans la production totale du continent. Bien des pays africains ayant adopté des programmes d'ajustement structurel n'enregistrent toujours qu'une croissance fort limitée. Les investissements se font rares. Avec une croissance démographique d'environ $3 \%$, la modeste augmentation de $1 \%$ du PIB n'a de nouveau pas pu empêcher la dégradation du revenu réel par habitant.

16 La critique souvent adressée aux programmes d'ajustement structurel préconisés en Afrique par le FMI et la Banque mondiale et qui reposent sur la croissance des exportations, semble malheureusement confirmée par les chiffres. Il est vrai que les exportations de matières premières de ces pays augmentent en volume, mais à des prix 
réels toujours plus défavorables. Il existe quelques exceptions heureuses, tel le Ghana, mais ce pays est, avec l'Afrique du Sud, l'un des rares exportateurs d'or du continent. La chute des prix des matières premières et des produits agricoles éprouve ces pays, et le protectionnisme agricole des pays industrialisés rend la commercialisation des produits africains plus difficile (exemple : il est perçu sur le chocolat africain une taxe douanière environ cinq fois supérieure à celle perçue sur le cacao africain. NZZ, 7.6.93).

Les investissements directs nets (entrées moins sorties) dans les pays en développement ont encore augmenté par rapport à 1991. Selon le FMI, ils ont passé d'environ 30 milliards de dollars en 1991 à 38 milliards de dollars en 1992. Ce sont les pays d'Asie qui en ont absorbé la plus grande part (18 milliards de dollars net), mais les investissements en Amérique latine et en Europe de l'Est ont également sensiblement augmenté (respectivement 12 milliards de dollars et 3 milliards de dollars). Il semble que la libéralisation du commerce, les privatisations et la dérégulation des marchés ainsi que l'ouverture accrue au commerce extérieur aient favorisé les investissements directs dans les pays en développement. Au niveau mondial, les investissements directs ont atteint en 1990 leur maximum de 234 milliards de dollars pour redescendre à 183 milliards de dollars en 1991 et 159 milliards de dollars en 1992 (selon le Rapport de la CNUCED de 1993 sur les investissements dans le monde). Cette évolution s'explique surtout par la récession des pays industrialisés.

22 L'OCDE et la Banque mondiale signalent que le transfert net des ressources a connu en 1992 une évolution très favorable aux pays en développement. Malgré la récession des pays industrialisés, le flux total de capitaux dans les pays en développement a augmenté en 1992 de $20 \%$ par rapport à 1991. Les chiffres de la Banque mondiale sont récapitulés dans le tableau $n^{\mathrm{e}} 2$. Pour la première fois, les moyens privés l'ont emporté sur les moyens publics, surtout en raison de la forte augmentation des prêts bancaires privés et de l'augmentation des investissements directs. Le flux des crédits privés a atteint, avec 44 milliards de dollars, son point le plus haut des dix dernières années.

Annuaire suisse de politique de développement, 13 | 1994 
Quant à l'aide publique au développement, elle n'a connu qu'un modeste développement ces dix dernières années. En 1992, l'APD a même reculé.

Tableau $\mathrm{N}^{\circ}$ 2. Apport des ressources vers les pays en développement

(en milliards \$)

\begin{tabular}{|lr|r|r|r|r|}
\hline & 1989 & 1990 & 1991 & 1992 & $1^{1993}$ \\
\hline Aide publique au développement & 41,1 & 59,1 & 62,9 & 54,6 & 63,5 \\
$\quad$ Dons & 19,0 & 28,5 & 32,9 & 34,5 & 35,5 \\
Crédits & 22,1 & 30,7 & 30,0 & 20,1 & 27,9 \\
Crédits privés & 10,2 & 12,9 & 13,8 & 41,7 & 43,7 \\
Investissements directs $_{\text {Investissements de portefeuille }}$ & 24,7 & 26,3 & 36,9 & 47,3 & 56,3 \\
Apport net de ressources $^{2}$ & 3,5 & 3,8 & 7,6 & 13,1 & 13,2 \\
Transfert net de ressources $^{3}$ & 79,5 & 102,1 & 121,1 & 156,6 & 176,7 \\
& 2,3 & 25,5 & 44,5 & 79,6 & 91,6 \\
\hline
\end{tabular}

Source : World Debt Tables 1993-94, in : NZZ, 16.12.1993.

1) des chiffres pronostiques.

2) Apport de capital moins les amortissements

3) Apport net de ressources déduit des intérêts et des transferts de bénéfices.

\section{Importantes différences régionales}

L'importance des écarts régionaux en matière de développement économique est de nouveau flagrante. L'évolution observée tout au long des années 80 se poursuit: une croissance marquée en Asie, modeste et accompagnée d'une baisse du revenu par habitant en Afrique comme en Amérique latine. La distribution du revenu mondial n'est pas plus équitable que naguère, les plus pauvres n'ont pas vu leur part augmenter. Une enquête de la Banque des règlements internationaux (63ème rapport annuel, Bâle, 1993, p. 69) montre que le $20 \%$ le plus pauvre de l'humanité disposait en 1960 de $5 \%$ du revenu mondial, et que cette part est tombée à $3,4 \%$ en 1992. En Afrique, les revenus n'ont fait que se dégrader au cours de cette décennie; le revenu de l'Amérique latine, stable au début des années 80 , a sensiblement diminué. Aujourd'hui, bien des gens doutent dans le monde que l'écart entre pays pauvres et pays développés puisse être réduit par des transferts de technologies et des transferts de capitaux, ou que la manne du développement filtre automatiquement jusqu'aux couches les plus démunies de la population.

\section{Rapport du PNUD sur le développement}

Depuis quatre ans, le Programme des Nations Unies pour le développement (PNUD) publie un rapport annuel sur le développement humain qui ajoute aux indicateurs habituels des institutions financières (dérivés uniquement des produits nationaux bruts ou des revenus réels par habitant) un "indicateur du développement humain" multidimerisionnel. Le rapport 1993 signale que la croissance enregistrée dans les pays industrialisés et les pays en développement ne s'accompagne pas de la création d'emplois, ce qui provoque le chômage total ou partiel de millions de personnes de par le monde. Les auteurs plaident pour une "nouvelle répartition de la croissance" : il s'agit de réévaluer le facteur travail humain en renforçant les investissements dans la formation et la santé, en particulier chez les femmes. Le facteur capital doit être élargi aux dimensions du "capital humain" et du "capital environnemental". Il faut 
encourager les technologies à forte main-d'œuvre et, dans les pays en développement, l'emploi dans le secteur informel. Le rapport plaide d'autre part pour la décentralisation économique et politique. Le capital est beaucoup trop concentré dans les centres.

Tableau $\mathrm{N}^{\circ}$ 3. Répartition du revenu par régions

\begin{tabular}{|c|c|c|c|c|}
\hline \multirow[t]{2}{*}{ Région / Pays } & 1960 & 1970 & 1980 & 1990 \\
\hline & \multicolumn{4}{|c|}{$\begin{array}{l}\text { Revenu par habitant par rapport à } \\
\text { la moyenne des pays industriels }\end{array}$} \\
\hline Amérique latine & 0,41 & 0,38 & 0,40 & 0,29 \\
\hline Afrique & 0,17 & 0,14 & 0,14 & 0,11 \\
\hline Asie & 0,25 & 0,26 & 0,31 & 0,35 \\
\hline Chine & 0,14 & 0,12 & 0,13 & 0,20 \\
\hline NEI & 0,31 & 0,40 & 0,59 & 0,83 \\
\hline \multirow[t]{2}{*}{ Ensemble des pays en développement } & 0,25 & 0,24 & 0,24 & 0,20 \\
\hline & \multicolumn{4}{|c|}{$\begin{array}{l}\text { Croissance moyenne du revenu } \\
\text { par habitant } \\
\text { au cours des dix années précédentes }\end{array}$} \\
\hline \multicolumn{5}{|l|}{ Pour mémoire: } \\
\hline Amérique latine & - & 3,0 & 3,1 & $-0,8$ \\
\hline Afrique & - & 2,2 & 2,0 & $-0,3$ \\
\hline Asie & - & 3,5 & 3,4 & 2,7 \\
\hline Chine & - & 2,2 & 3,5 & 6,9 \\
\hline NEI & - & 6,3 & 6,7 & 5,6 \\
\hline Ensemble des pays en développement & - & 2,9 & 2,4 & $-0,1$ \\
\hline
\end{tabular}

Sources : Summers, R. und Heston, A. (1991) : "The Penn World Table (Mark 5) : an expanded set of international comparisons, 1950-88", Quarterly Journal of Economies, S. 327-348; IMF, International Financial Statistics; World Bank, World Tables ; estimations BRI, dans : Rapport annuel BRI, Bâle, 1993.

NOTE : Les calculs sont établis à partir de moyennes par pays et ne prennent pas en compte les différences de revenu au sein du pays.

\section{BIBLIOGRAPHIE}

Banque des règlements internationaux, 63ème rapport annuel, Bâle, juin 1993

PNUD, Rapport mondial sur le développement humain, 1993

CNUCED, World Investment Report 1993, commenté dans : NZZ 21.7.1993

Bulletin CS 5-6/93, Rohwarenmärkte und Entwicklungsländer et Entwicklungsländer als Konjunkturlokomotive?

OCDE, Intégration régionale et pays en développement, Paris 1993 
OCDE, Communiqués de presse, 9.7.1993 (investissements directs étrangers) ; mars 1993 (investissements en Amérique latine)

GATT-Focus n 98, avril 1993 (commerce mondial 1992)

IMF-Survey, mai 1993 (World Economie Outlook) ; juin 1993 (l'économie asiatique en chiffres, rapport $\mathrm{ADB}$ )

NZZ, 13.4.1993 (les investissements étrangers en Amérique latine)

NZZ, 13.4.1993 (accords commerciaux en Asie du Sud-Est)

NZZ, 14.4.1993 (rapport de la commission économique de l'ONU pour l'Europe (CEE)/ l'économie de l'Europe de l'Est en chiffres)

NZZ, 21.4.1993 et 4.6.1993 (l'économie de la Chine en chiffres)

NZZ, 7.6.1993 (rapport annuel BAD / l'économie africaine en chiffres)

NZZ, 2.7.1993 et 12.7.1993 (accord de libre-échange ALENA)

NZZ, 9.7.1993 (sommet économique du G-7)

NZZ, 21.7.1993 (CNUCED, Rapport sur les investissements dans le monde) Tages-Anzeiger, 16.1.1993 (étude Africa and World Trade)

\section{NOTES}

1. En l'absence d'autres indications, les données économiques de ce chapitre sont tirées du 63ème rapport annuel de la Banque des règlements internationaux (Bâle, juin 1993).

\section{AUTEURS}

\section{MONIKA EGGER}

Chargée de recherche, IUED

\section{GÉRARD PERROULAZ}

Chargé de recherche, Université de Genève et IUED

\section{RENÉ LONGET}

(avec la participation de...), Directeur romand de la Société suisse pour la protection de l'environnement 